\title{
Stress Modelling Using Transfer Learning in Presence of Scarce Data
}

Conference Paper · December 2015

DOI: 10.1007/978-3-319-26508-7_22

CITATIONS

2

6 authors, including:
READS

39
Pablo Hernandez-Leal

Centrum Wiskunde \& Informatica

28 PUBLICATIONS 74 CITATIONS

SEE PROFILE

Eduardo F Morales

Instituto Nacional de Astrofísica, Óptica y Elect. .

210 PUBLICATIONS 1,255 CITATIONS

SEE PROFILE

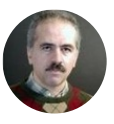

Luis Enrique Sucar

Instituto Nacional de Astrofísica, Óptica y Elect...

310 PUBLICATIONS $\quad 2,000$ CITATIONS

SEE PROFILE

Oscar Mayora

FBK CREATE-NET

117 PUBLICATIONS 650 CITATIONS

SEE PROFILE

Some of the authors of this publication are also working on these related projects: 


\title{
Stress Modelling Using Transfer Learning in Presence of Scarce Data
}

\author{
Pablo Hernandez-Leal ${ }^{1}$, Alban Maxhuni ${ }^{2}$, L. Enrique Sucar ${ }^{1}$, Venet Osmani ${ }^{2}$, \\ Eduardo F. Morales ${ }^{1}$, Oscar Mayora ${ }^{2}$ \\ 1 Instituto Nacional de Astrofísica, Óptica y Electrónica \\ Sta. María Tonantzintla, Puebla, México \\ \{pablohl, esucar, emorales\}@ccc.inaoep.mx \\ 2 CREATE-NET \\ Via alla Cascata 56/D Povo - Trento, Italy \\ \{amaxhuni, vosmani, omayora\}@create-net.org
}

\begin{abstract}
Stress at work is a significant occupational health concern nowadays. Thus, researchers are looking to find comprehensive approaches for improving wellness interventions relevant to stress. Recent studies have been conducted for inferring stress in labour settings; they model stress behaviour based on non-obtrusive data obtained from smartphones. However, if the data for a subject is scarce, a good model cannot be obtained. We propose an approach based on transfer learning for building a model of a subject with scarce data. It is based on the comparison of decision trees to select the closest subject for knowledge transfer. We present an study carried out on 30 employees within two organisations. The results show that the in the case of identifying a "similar" subject, the classification accuracy is improved via transfer learning.
\end{abstract}

Keywords: Stress modelling, Transfer learning, Semi-supervised learning

\section{Introduction}

Over the last four decades there has been rising concern in many countries about the growth and consequences of work-relevant stress and burnout. Recent reports show that stress is ranked as a second most common work-related health problem across the members of the European Union [1]; the same report shows that individuals with high levels of stress were accompanied by physical and psychosocial complaints and decreased work-control for the requirements placed on them.

To date, current approaches to measuring stress rely on self-reported questionnaires [2], which can be a source of subjectivity. As such, the availability of rich set of embedded sensors in smartphones is increasingly being used to provide objective measures of behaviour phenomena.

The objective of this study was to model stress levels from different behavioural variables obtained from smartphones when the labelled data for a 
person is scarce. Collected data includes information related to psychological self-assessments that are obtained from standardised validated questionnaire and the sensor data from smartphones from 30 employees in two different organisations. Our approach learns a model for each subject which is useful not only to predict but to perform comparisons among different subjects in order to obtain groups of people (clusters) that behave similarly. When a model is built for a new subject it usually contains insufficient information to have an accurate model. For this reason we use a transfer learning approach that uses data from the most similar user in order to improve the model which results in better accuracy.

We performed an experimental analysis using real data focused on predicting stress based on several smartphone sensors. The study includes 3 aspects: (i) using semi-supervised learning to complete the models for subjects with missing data, (ii) clustering the subjects based the similarity of the learned decision trees, (iii) applying transfer learning to try to improve the model of a new user with scarce data.

\section{Related Work}

Smartphones have already been used to detect mental health conditions, as shown in our previous work 3 . However, capturing what is causing an emotion change that is influenced from work-related stressors and detect the onset of stress can be quite challenging. Current methods have tried to infer stress based on physiological signals, e.g., heart-rate variability, blood pressure, body temperatures and respiration [7. Furthermore, recent work emphasize the importance of measuring physiological signals that would help providing short-term feedback to the users in everyday activities 8. However, these methods have as drawback that they need to be carried at all times (and in specific places in body) in order to allow accurately and continuous monitoring.

StressSense 9 proposes a method for detecting stress based on speech analysis and the variation of speech articulation. However, in real-life activities (e.g., crowded environments) this approach may lead to misinterpretation of speech and therefore of emotion.

In order to infer relation dynamics of people and behaviour changes in daily activities, smartphones have been suggested as a promising candidate to obtain user's context. Research using smartphones for long-term monitoring [10 have reported that smartphone sensing can be used to collect many types of contextual data including: physical activities, body postures and locations. In 11, the authors build a self-tracking system called MoodScope to help its users manage their mood. The system detects users mood from smartphones usage data (email, call and SMS logs, application usage, web history and location changes). The authors reported their initial $66 \%$ accuracy of 32 subjects from their daily mood and improving to $93 \%$ after two months of training.

Another relevant work is from Bauer et al. 12 whose work aimed at recognizing stress from 7 students before and after the exam period. The assumption is that students are likely to be under stress during the exam sessions. They acquired data from smartphones (location, social proximity through Bluetooth, 
phone calls and SMS logs) and they reported an average accuracy of $53 \%$ during the exam session. In recent work, Bogomolov et al.13 used call logs, SMS logs, proximity data, and self-reported surveys about personality traits. The authors reported detecting daily stress levels with a $72.28 \%$ accuracy combining real life data from different sources. However, measuring stress in uncontrolled settings poses several difficulties since it requires the efforts of human annotators about their current perceived stress and other variables relevant to stress.

Building an accurate model to predict stress requires a considerable amount of labelled data. However, users tend no to answer the questionnaires all the time, and for a new user we will have few samples. Hence, to overcome these issues, in this study we use semi-supervised learning methods that use unlabelled instances and combine the information in the unlabelled data with the explicit classification information of labelled data for improving the classification performance [14]. Another related technique used to improving accuracy when available data is limited is transfer learning [15]. To the best of our knowledge, no study has applied semi-supervised nor transfer learning methods aiming at predicting work-related stress.

\section{Data Acquisition}

For this study, we collected data from 30 healthy employee $3^{3}$ of two organisations located in the North-eastern part of Italy for a period of 8 weeks, further described in 3 . All subjects were given a smartphone ${ }^{4}$ where the application used for this study collected data continuously as a background application. The features we extracted for each subject are categorised into two types, the first group of variables includes information of user's behaviour that was collected from the smartphone sensors during work hours, these are called objective variables. The second group contains subjective information obtained from the self-reported questionnaires, that includes mood and work-relevant stress items.

\subsection{Self-reports}

The self-reported questionnaire included scales that have demonstrated validity for inferring stress (the Oldenburg Burnout Inventory [16]). The survey was administrated via the smartphones and the data obtained from the participants was obtained three times a day: 9:00am, 2:00pm, and 5:00pm, for a period of 8 weeks 5

In Table 1. we present the overall stress responses for the whole period (8 weeks), where we include only the questionnaires obtained from $(2: 00 \mathrm{pm}$ and

\footnotetext{
${ }^{3} 16$ male and 14 female; married (50\%) and not married (50\%); age ranged from $26-30(16.67 \%), 31-40(60 \%)$ and above $40(23.33 \%) ; 33.33 \%$ of participants had an academic degree, $36.7 \%$ had bachelor degree and $30 \%$ had high school education.

${ }^{4}$ Samsung Galaxy S3 mini 32GB.

${ }^{5}$ The scales ranged from 1-to-5 and items selection has been performed considering the specificity of the Italian work-context in order to ensure their suitability.
} 
Table 1: Overall number and percentage of Stress-responses

\begin{tabular}{|llcc|}
\hline Variable & Level & Nr.Responses (\%) & Nr.Subjects \\
\hline Perceived & High & $325(22.18 \%)$ & 27 \\
Stress & Moderate & $515(35.15 \%)$ & 30 \\
& Low & $625(42.66 \%)$ & 30 \\
\hline \multicolumn{2}{|c|}{ Total Responses: } & $\mathbf{1 4 6 5}(\mathbf{1 0 0 . 0 0 \% )}$ & $\mathbf{3 0}$ \\
\hline
\end{tabular}

5:00pm). The overall number of responses from both intervals was 1465 . In order to simplify the measurements of the work-related stress, we have classified the stress-level into three classes: $\leq 2$ as "Low-Stress", 3 as "Moderate-Stress", and $\geq$ 4 as "High-Stress". In Table 1 is shown that during the entire monitoring weeks, 27 -subjects have perceived "High-Stress" and is about $22.18 \%$ of the monitoring weeks.

Table 2: Number of parameters and features extracted from smartphones

\begin{tabular}{|c|c|c|}
\hline Category & $\begin{array}{l}\text { Smartphone } \\
\text { Sensors }\end{array}$ & Attributes (Feature-Extracted) \\
\hline 1. Physical Activity & Accelerometer & - 3-Axis (Magnitude) \\
\hline Level & & - 3-Axis (Variance Sum [1]) \\
\hline \multirow[t]{3}{*}{ 2. Location } & Cellular & - CellID and LACID (Number of clusters (DBSCAN) 18) \\
\hline & WiFi & - Access Points (Number of clusters (DBSCAN) 18]) \\
\hline & Google-Maps & $\begin{array}{l}\text { Latitude and Longitude (Number of clusters } \\
\text { (DBSCAN) 18, and distances 19) }\end{array}$ \\
\hline 3. Social Interaction & Microphone & - Proximity Interaction \\
\hline & & - Pitch 20, Mel-MBSES 21 \\
\hline \multirow[t]{7}{*}{ 4. Social Activeness } & Phone Calls & - Number of Incoming and Outgoing Calls \\
\hline & & $\begin{array}{l}\text { - Duration of Incoming and Outgoing Calls } \\
\text { - Most common Contact-Calls }\end{array}$ \\
\hline & SMS & - Number of Incoming and Outgoing SMS \\
\hline & & - Duration of Incoming and Outgoing SMS \\
\hline & Calendars & - Most common Contact-SMS \\
\hline & App usage & - Number of used applications (Social, System) \\
\hline & & - Duration of used applications (Social, System) \\
\hline
\end{tabular}

\subsection{Objective Data Acquisition and Features Extraction}

In Table 2 we provide an overview of the types of smartphone data acquired for the study. In addition, we provide an overview of each category used in our study:

- Physical Activity Level: We measured the level of activity using accelerometer data capturing 3-axial linear acceleration continuously at a rate of $5 \mathrm{~Hz}$, which was sufficient to infer physical activity levels. For extracting features, we used the method developed in the framework in [17] and we measured the magnitude and the variance sum of 26 seconds [not clear] ( $n=128$-samples) from accelerometer readings. Each segment was classified into "high", "low", and "none" activity levels.

- Location: Location patterns and the location changes were measured using the list of WiFi access points (AP) available with their respective BSSID address, cell tower location and Google location information (latitude, longitude). We performed clustering for WiFi by means of the received signal strength (RSS) from each access point (AP). Density-based clustering (DBSCAN) 18 was used to obtain a number of different locations (clusters) in 
hourly basis. Similarly, DBSCAN was used to cluster Google location and cellular tower location.

- Social Interaction: We have used the microphone sensor embedded on smartphones in order to capture verbal interaction within the employees when they where involved in conversation in a close proximity. We have extracted two main audio features (Pitch $\mid 20$ and Mel-MultiBand Spectral Entropy Signature (Mel-MBSES) [21]) to perform speech recognition. From the classifier, we obtain the values (true, false) and we measure the distribution of the interactivity in a daily basis.

- Social Activeness: We have included measurements from the phone usage (number and duration of phone calls, number and length of SMS messages) and the usage of two types of apps: system (Camera, Calendar, Webbrowsing, Mail) and social (WhatsApp, Facebook, Skype) that were installed on the smartphones.

\section{Learning and Comparison of Models}

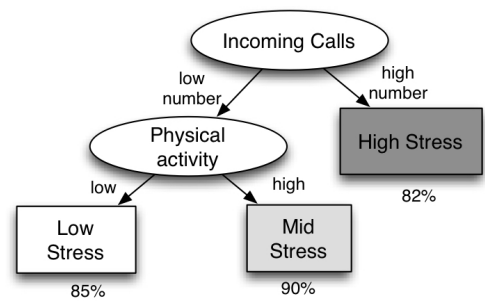

Fig. 1: Decision tree that classifies the level of Stress of one of the subjects in the study. Ovals represent decision nodes. Rectangles are leaves (terminal nodes) that give the classification value, in this case they represent low, mid or high level of stress.

Predicting perceived stress of the user can be modelled as a classification problem. We used decision trees [22] to model subject's stress since this representation can be easily understood by a human, and this could help to have a better understanding of what causes stress. Also, using this representation we can compare the models for the different subjects, which is important for transfer learning. Our approach is to build a decision tree (can be seen as a model) to predict stress for each subject of the study. To learn decision trees we used the C4.5 algorithm using as attributes the objective variables presented in Section 3.2. The class to predict is the self-reported stress level (Section 3.1) (Low, Mid, High). In Fig. 1 we depict an example of a decision tree classifying the stress level of a subject.

Our first objective is to analyse how subjects are related to each other in terms of how similar are their models. To compare the trees, the dissimilarity measure presented in 23 is used. This measure combines the structure (the 
attributes of the nodes) and predictive (the predicted classes) similarities in a single value. The dissimilarity measure among two trees $T_{i}$ and $T_{j}$ is defined as ${ }^{6}$

$$
d\left(T_{i}, T_{j}\right)=\sum_{h=1}^{H} \alpha_{h}\left(1-s_{h}\right) \frac{m_{h 0}}{n}+\sum_{k=1}^{K} \alpha_{k}\left(1-s_{k}\right) \frac{m_{0 k}}{n}
$$

where the $m_{x y}$ values measure the predictive similarity and the $\alpha_{x}$ and $s_{x}$ values measure the structural similarity. This measure can be normalized to be in the range $[0-1]$, where 0 represents that the trees are very similar and 1 that they are totally dissimilar.

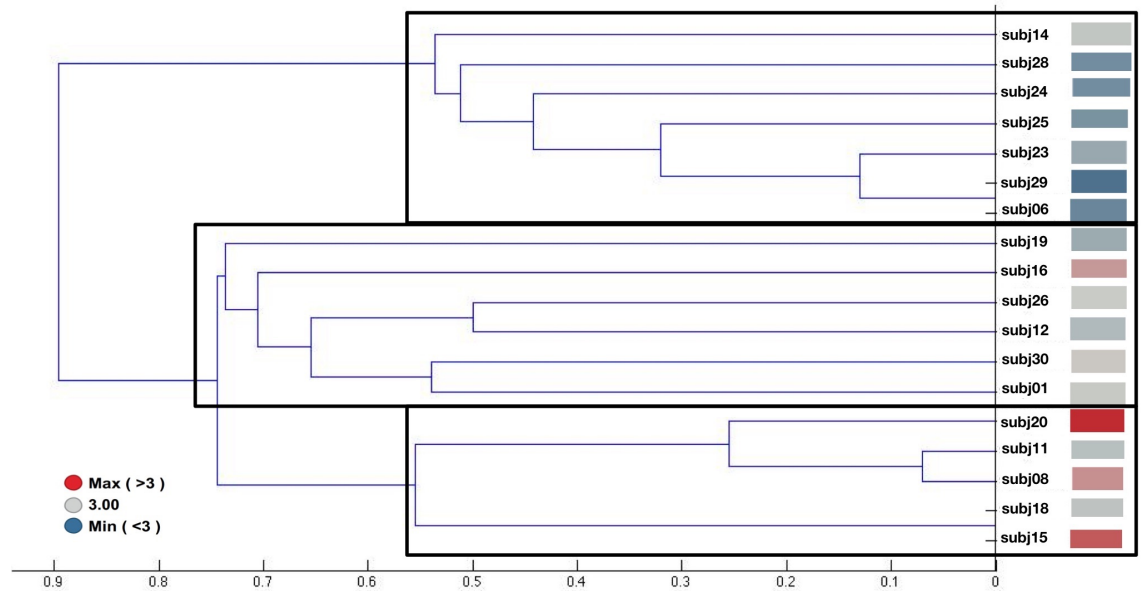

Fig. 2: Dendrogram obtained by computing similarities between models of each subject (using only 18 subjects). Three major clusters can be noted, colour boxes correspond to average stress for different subjects.

\subsection{Initial dataset}

From the set of 30 subjects, we initially removed those that had a significant number of missing values (mainly in the questionnaires for self-evaluation of their stress level). Thus, having a remaining set of 18 subjects.

A decision tree was learned for each subject and using the distance in Equation (1) we compared all pairs of models to obtain a similarity matrix. From that matrix we performed hierarchical clustering using the unweighted pair group method with arithmetic mean algorithm which yields the dendrogram depicted in Fig. 2, where a box with a colour indicates the average self-reported stress for that subject. From the figure we can observe 3 clusters with 7,6 and 4 subjects. The largest cluster (with 7 subjects) roughly corresponds to subjects which reported low levels of stress in average (denoted by the blue boxes). The second

${ }^{6}$ Let $1, \ldots, H$ as the leaves of $T_{i}$, and $1, \ldots, K$ as the leaves of $T_{j}, m_{h k}$ is the number of instances which belong to both the $h$ th leaf of $T_{i}$ and to the $k$ th leaf of $T_{j}$. 
major cluster (with 6 subjects) corresponds to subjects who reported a mid level of stress (grey boxes). The third cluster with only 4 subjects shows subjects with high and mid level of stress.

Since the initial data had a large portion of missing values $(20.03 \%$ of overall dataset), semi-supervised learning was used to fill those. In this study, we use a single classifier method called self-training [14 to classify the unlabelled data. These new data is added to the training set, the classifier is re-trained and the procedure repeated.

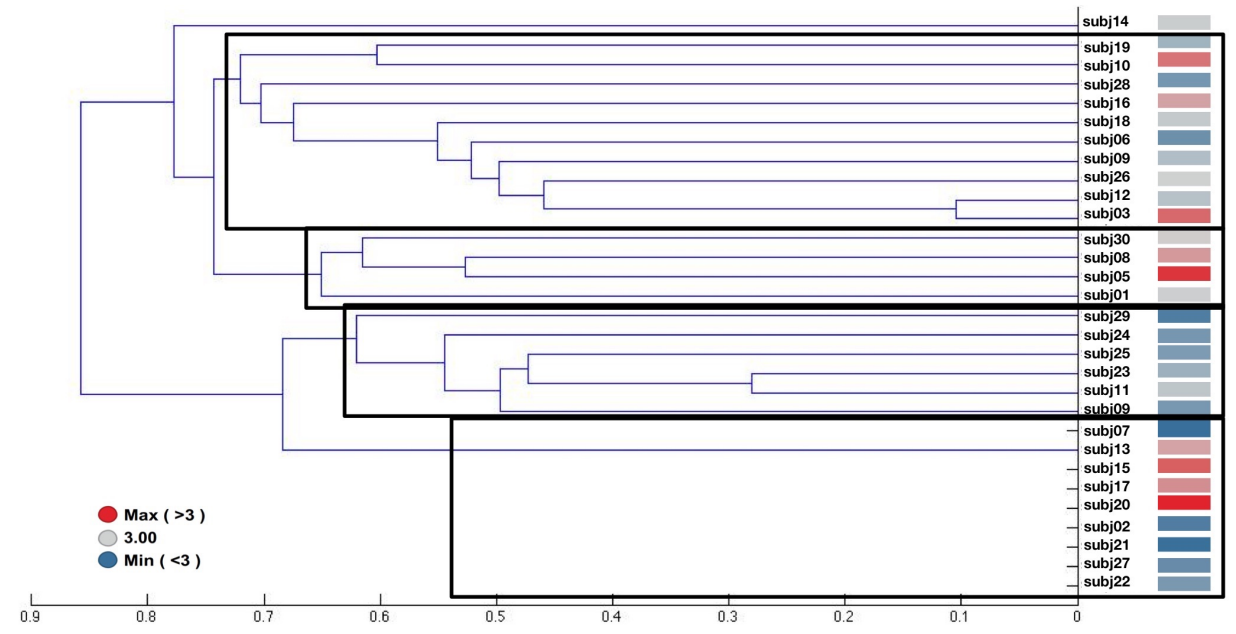

Fig. 3: Dendrogram obtained by computing similarities between models of each subject (for the 30 subjects) after using semi-supervised learning to fill missing data. Colour boxes correspond to average stress for each subject.

After applying the semi-supervised learning phase, there is enough data to compute comparisons with the 30 users of the study. A dendrogram of the similarity of all users is depicted in Fig. 3. In this case we can observe 4 main clusters. One cluster (bottom, 9 subjects) is formed by those subjects whose stress level was consistently the same for the entire study. Note, that in this case subjects were clustered by the rule of behaviour (always the same stress level) not by the prediction itself. Another cluster is formed by subjects that on average showed low and mid level of stress (6 subjects). A third cluster (with 4 subjects) contains subjects with high and mid levels of stress. The largest cluster is formed with 10 subjects which do not show a consistent level of stress.

\subsection{Similarity matrices}

The process described in previous section was used to obtain a similarity matrix, depicted in Fig. 4 (a), where the more similar a subject is to another the more darker that square is (subjects are ordered by clusters). Then we decided to remove $50 \%$ of the data from all subjects (this will be useful for our transfer 
learning approach in the next section). The similarity matrix using this reduced data is depicted Fig. 4(b), and in Fig. 4 (c) we depict the matrix resulting from the difference of (a) and (b) where a grey value means no difference.

In summary, we have three similarity matrices with different quantity of data. For each matrix we computed the average values, the initial data with 18 subjects showed a more disperse set of distances with an average of $0.65 \pm 0.18$ (higher value, means subjects are more different to each other). After the semisupervised algorithm was applied the average distance was $0.55 \pm 0.16$ even when number of subjects increased (30 subjects). Finally, when the data was reduced the average distance decreased to $0.49 \pm 0.15$, which may not happen in all cases (note that removed data was randomly selected).

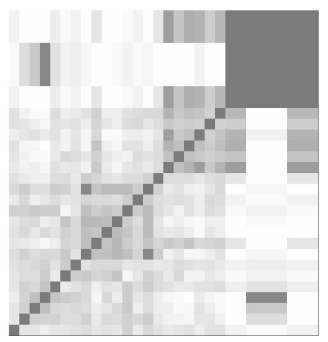

(a)

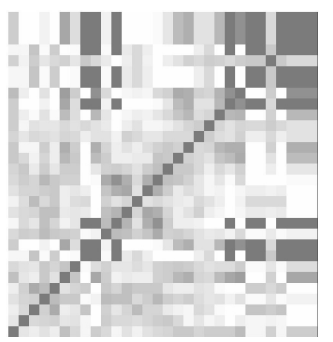

(b)

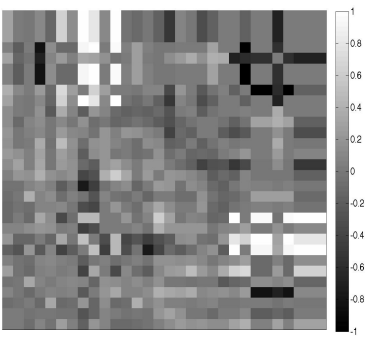

(c)

Fig. 4: Similarity matrices of 30 users using (a) all data (after semi-supervised learning) and (b) with 50\% of instances removed -darker cells indicate high similarity. (c) depicts the difference between (a) and (b); a white cell indicates $\mathrm{a}+$ difference, black a - negative difference, and grey no difference.

\subsection{Missing data and semi-supervised learning}

Table 3: Percentage of entries whose value increased more than $\epsilon=0.1, \ldots, 0.5$ from matrix original to matrix modified.

\begin{tabular}{|l|l|l|l|l|l|l|l|}
\hline \multicolumn{2}{|c|}{ Data } & \multicolumn{4}{c|}{$\Delta$ (Original, Modified $)>\epsilon$} \\
\hline Original & Modified & $\epsilon=0.1$ & $\epsilon=0.2$ & $\epsilon=0.3$ & $\epsilon=0.4$ & $\epsilon=0.5$ \\
\hline Initial & Semi-supervised added data & 0.39 & 0.22 & 0.14 & 0.07 & 0.05 \\
Semi-supervised & Removed random 50\% data & 0.55 & 0.29 & 0.19 & 0.13 & 0.11 \\
\hline
\end{tabular}

Now we analyse the effect of adding/removing data in terms of the comparisons among models, i.e. similarity matrices. Thus, we computed the absolute difference of each entry between an original and a modified matrix:

$$
\Delta i, j(\text { original }, \text { modified })=\left|e_{i, j}^{\text {original }}-e_{i, j}^{\text {modified }}\right|
$$

Table 3 shows the percentage of entries where $\Delta_{i, j}>\epsilon$ with $\epsilon=0.1, \ldots, 0.5$ between two matrices. After applying the semi-supervised approach, $39 \%$ of the entries in the similarity matrix changed more than 0.1 , which is a small change 
in similarity for a large portion of entries. In contrast only $5 \%$ of entries changed more than 0.5. Results show that after the semi-supervised approach the similarity matrices were slightly altered with an average value of $0.12 \pm 0.14$, meaning there were no drastic changes in similarities. When we reduced the data by $50 \%$ the difference between matrices increased to $0.19 \pm 0.20$ which is expected since the data was reduced significantly. These results are important to show that the similarity used is robust to even when there is missing values. This will be useful in the next section since we start with the reduced data but using transfer learning we can improve their accuracy.

\section{Transfer Learning}

Our approach assumes a set of previously learned models along with their respective data. Then, a new subject appears; however, its associated data is scarce which results in having a model with poor predictive accuracy. Information from the other subjects could be useful to improve the model. First we learn a model $t_{i}$ for the new subject $i$ and compare with the rest $T$ using equation 1 . The model $k=\operatorname{argmin}_{t_{j} \in T} d\left(t_{i}, t_{j}\right)$ which is the most similar to $t_{i}$ is selected and its data is transferred to $i$. A new model is learned using the original and the transferred data.

We applied the described approach on the data which has a percentage of data removed. The average distance to the nearest subject is 0.28 and there are 18 subjects that have only one nearest subject. These subjects were selected for the proposed transfer learning approach.

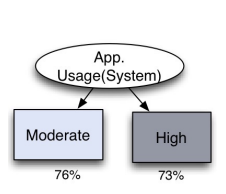

(a)

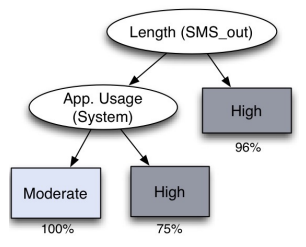

(b)

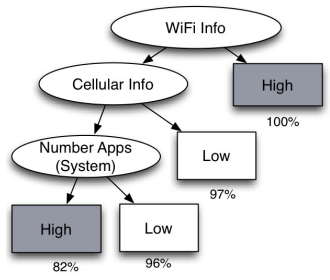

(c)

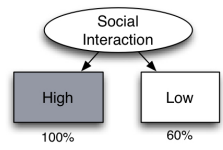

(d)

Fig. 5: Learned models of different subjects: $\operatorname{Subj30}$ (a) and its most similar Subj17 (b). Subj29 (c) and its most similar model Subj05 (d).

We compare the results using the reduced data with and without the transfer learning approach to classify the same data. Accuracy is obtained by learning a classifier using either: the reduced data or the reduced plus transferred data, then testing that model on the data without removed instances. The results showed that using transfer did not improve the accuracy for all subjects. This happens because there is information we are ignoring of when transfer will be more useful: the distance to the nearest subject. The idea is to use transfer when the distance is small (when the model is close to another ) and not when distance increases. For example, in Fig. 5 (a) and (b) we depict trees that of subjects 30 and 17 which have a $d=0.36$. In this case trees are similar in their decision 
Table 4: Classification accuracy, $\Delta$ transfer shows the difference between no transfer and transfer columns. All data shows the accuracy using all original data (upper bound). The number of initial and transferred instances is shown.

The top part of the table shows the results when the distance to the closest

subject is small $(<0.37)$, while the bottom when it is large $(>0.37)$.

\begin{tabular}{|l|l|l|l|l|c|c|c|}
\hline Subject & No Transfer & Transfer & All data & $\Delta$ Transfer & Initial I. & Transf. I. & d(nearest) \\
\hline Subj 28 & 57.35 & 63.23 & 77.94 & 5.88 & 35 & 26 & 0.18 \\
Subj10 & 44.89 & 51.02 & 71.42 & 6.13 & 26 & 31 & 0.27 \\
Subj 12 & 55.93 & 54.23 & 62.71 & -1.69 & 18 & 31 & 0.32 \\
Subj18 & 70.27 & 62.16 & 75.67 & -8.10 & 31 & 18 & 0.32 \\
Subj24 & 67.14 & 67.14 & 71.42 & 0.00 & 36 & 31 & 0.36 \\
Subj05 & 70.68 & 65.51 & 86.20 & -5.17 & 29 & 37 & 0.36 \\
Subj30 & 42.85 & 53.57 & 78.57 & 10.71 & 37 & 29 & 0.36 \\
Subj09 & 57.69 & 73.07 & 76.92 & 15.38 & 18 & 35 & 0.36 \\
\hline Average & 58.35 & $\mathbf{6 1 . 2 4}$ & 75.10 & 2.89 & 28.75 & 29.75 & 0.31 \\
\hline Subj25 & 85.71 & 83.67 & 89.79 & -2.04 & 24 & 31 & 0.39 \\
Subj04 & 81.25 & 71.88 & 84.37 & -9.38 & 32 & 31 & 0.42 \\
Subj08 & 57.41 & 50.00 & 55.55 & -7.41 & 27 & 35 & 0.46 \\
Subj16 & 61.11 & 62.96 & 74.07 & 1.85 & 30 & 29 & 0.48 \\
Subj14 & 51.56 & 48.44 & 82.81 & -3.13 & 32 & 31 & 0.49 \\
Subj23 & 53.33 & 50.00 & 58.33 & -3.33 & 32 & 35 & 0.53 \\
Subj19 & 60.00 & 53.33 & 90.00 & -6.67 & 33 & 26 & 0.54 \\
Subj01 & 72.86 & 61.43 & 78.57 & -11.43 & 41 & 32 & 0.58 \\
Subj29 & 62.07 & 44.83 & 79.31 & -17.24 & 29 & 33 & 0.60 \\
Subj11 & 65.45 & 74.55 & 72.72 & 9.09 & 30 & 29 & 0.62 \\
\hline \hline Average & $\mathbf{6 5 . 0 8}$ & 60.11 & 76.55 & -4.97 & 31 & 31.2 & 0.51 \\
\hline
\end{tabular}

nodes. In contrast we show models of subjects 29 and 5 (in Fig. 5 (c) and (d)) which have $d=0.60$, here these trees show different decision nodes.

Table 5: Classification accuracies with different threshold for applying transfer learning and percentage of instances with transfer approach.

\begin{tabular}{|l|l|l|}
\hline Transfer threshold & \% Transferred Instances & Average Accuracy \\
\hline 0.00 & 0.00 & 62.09 \\
0.30 & 0.11 & 62.76 \\
0.37 & 0.44 & $\mathbf{6 3 . 3 7}$ \\
0.50 & 0.72 & 62.26 \\
1.00 & 1.00 & 60.61 \\
\hline
\end{tabular}

Using transfer only when distance was less than a transfer threshold (in this case $<0.37$ ) improves the accuracy from 58.35 to 61.24 (Table 4), in contrast when $d \geq 0.37$ is better not use transfer learning since the models are far from each other and this causes a negative transfer. We performed experiments varying the threshold with values $0.0,0.3,0.37,0.5,1.0$, average accuracy for all subjects is shown in Table 5. From the results we can see that the trivial approaches of not using transfer or using transfer on all subjects do not obtain the best results. However, selecting the appropriate threshold of transfer increases the accuracy. 
Finally, we also performed experiments removing only $20 \%$ of the data and repeated the transfer learning process. However, the transfer learning approach did not improve the accuracy. Preliminary analysis show that transferring all the data from the closest user is not always the best choice, a selection of the data must be applied and we leave that as future work.

\section{Conclusion}

Using smartphones to predict the affective state of a person, such as stress, requires a considerable amount of data to build a user-specific model. However, having enough labelled data is difficult. In this paper we have proposed an approach that combines semi-supervised and transfer learning to deal with this issue. An experimental evaluation was conducted with 30 subjects to predict stress at work. The results show that transfer from similar subjects can improve the classification accuracy, but using transfer from dissimilar persons could be detrimental. Future research ideas are to select instances of data to transfer from several similar models.

\section{Acknowledgements}

The work on this paper was partially funded by EC Marie Curie IRSES Project UBIHEALTH - 316337.

\section{Bibliography}

[1] Agence européenne pour la sécurité et la santé au travail, Malgorzata Milczarek, Eusebio Rial-González, and Elke Schneider. Occupational safety and health in figures: stress at work-facts and figures. Office for Official Publications of the European Communities, 2009.

[2] Petri Näätänen and Veijo Kiuru. Bergen burnout indicator 15. Edita, 2003.

[3] E. Ceja, V. Osmani, and O. Mayora. Automatic stress detection in working environments from smartphones' accelerometer data: A first step. Biomedical and Health Informatics, IEEE Journal of, PP(99):1-1, 2015.

[4] Venet Osmani. Smartphones in Mental Health: Detecting Depressive and Manic Episodes. Pervasive Computing, IEEE, 14(3):10-13, 2015.

[5] Raihana Ferdous, Venet Osmani, and Oscar Mayora. Smartphone app usage as a predictor of perceived stress levels at workplace. IEEE, 82015.

[6] A Grunerbl, A Muaremi, V Osmani, G Bahle, S Ohler, G Troster, O Mayora, C Haring, and P Lukowicz. Smartphone-Based Recognition of States and State Changes in Bipolar Disorder Patients. Biomedical and Health Informatics, IEEE Journal of, 19(1):140-148, 2015.

[7] Jorn Bakker, Mykola Pechenizkiy, and Natalia Sidorova. What's your current stress level? detection of stress patterns from gsr sensor data. In Data Mining Workshops, 2011 IEEE 11th International Conference on, pages 573-580. IEEE, 2011.

[8] Karen Kay-Lynn Liu. A personal, mobile system for understanding stress and interruptions. Master's thesis, MIT Media Arts and Science, 2004. 
[9] Hong Lu, Denise Frauendorfer, Mashfiqui Rabbi, Marianne Schmid Mast, Gokul T Chittaranjan, Andrew T Campbell, Daniel Gatica-Perez, and Tanzeem Choudhury. Stresssense: Detecting stress in unconstrained acoustic environments using smartphones. In Proceedings of the 2012 ACM Conference on Ubiquitous Computing, pages 351-360, 2012.

[10] R. Kocielnik, N. Sidorova, F.M. Maggi, M. Ouwerkerk, and J.H.D.M. Westerink. Smart technologies for long-term stress monitoring at work. In Computer-Based Medical Systems, 2013 IEEE 26th International Symposium on, pages 53-58, 2013.

[11] Robert Likamwa, Yunxin Liu, Nicholas D Lane, and Lin Zhong. Moodscope: building a mood sensor from smartphone usage patterns. In Proceeding of the 11th annual international conference on Mobile systems, applications, and services, pages 389-402, 2013.

[12] Gerald Bauer and Paul Lukowicz. Can smartphones detect stress-related changes in the behaviour of individuals? In Pervasive Computing and Communications Workshops (PERCOM Workshops), 2012 IEEE International Conference on, pages 423-426, 2012.

[13] Andrey Bogomolov, Bruno Lepri, Michela Ferron, Fabio Pianesi, and Alex Sandy Pentland. Daily stress recognition from mobile phone data, weather conditions and individual traits. In Proc. of the ACM International Conference on Multimedia, pages 477-486, 2014.

[14] Xiaojin Zhu. Semi-supervised learning. In Encyclopedia of Machine Learning, pages 892-897. Springer, 2010.

[15] Sinno Jialin Pan and Qiang Yang. A survey on transfer learning. Knowledge and Data Engineering, IEEE Transactions on, 22(10):1345-1359, 2010.

[16] Evangelia Demerouti and Arnold B Bakker. The oldenburg burnout inventory: A good alternative to measure burnout and engagement. Handbook of stress and burnout in health care. Hauppauge, NY: Nova Science, 2008.

[17] FUNF - Open Sensing Framework. http://funf.org/about.html, 2014.

[18] Derya Birant and Alp Kut. St-dbscan: An algorithm for clustering spatialtemporal data. Data \& Knowledge Engineering, 60(1):208-221, 2007.

[19] CC Robusto. The cosine-haversine formula. American Mathematical Monthly, pages 38-40, 1957.

[20] Per Hedelin and Dieter Huber. Pitch period determination of aperiodic speech signals. In Acoustics, Speech, and Signal Processing, 1990. ICASSP-90., 1990 International Conference on, pages 361-364. IEEE, 1990.

[21] Fredric J Harris. On the use of windows for harmonic analysis with the discrete fourier transform. Proceedings of the IEEE, 66(1):51-83, 1978.

[22] John Ross Quinlan. C4.5: programs for machine learning. Morgan Kaufmann, 1993.

[23] Rossella Miglio and Gabriele Soffritti. The comparison between classification trees through proximity measures. Computational Statistics and Data Analysis, 45(3):577-593, April 2004. 